\title{
CONHECIMENTO DOS MÉdICOS DA ATENÇÃO PRIMÁRIA À SAÚdE SOBRE RASTREAMENTO DE CÂNCER
}

PRIMARY HEALTH CARE PHYSICIANS' KNOWLEDGE ON CANCER SCREENING

CONOCIMIENTO DE MÉdiCOS DE LA ATENCIÓN PRIMARIA SOBRE RASTREO DE CÁNCER

Lorena Alves Trajano ${ }^{1}$

José Francisco Igor Siqueira Ferreira ${ }^{2}$

Miguel Marcelo Freire de Melo ${ }^{3}$

Luiz Eduardo de Castro Batista 4

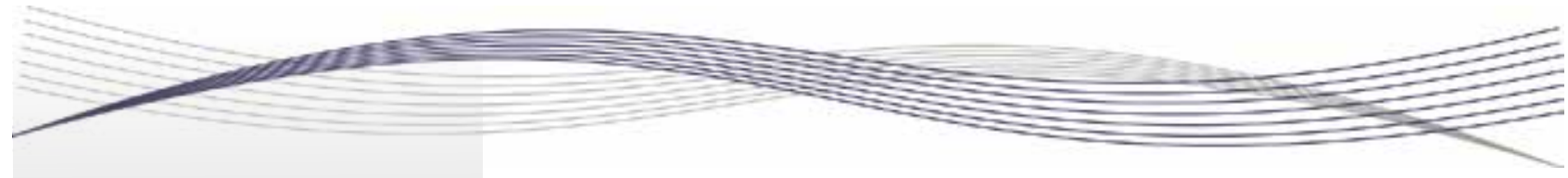

Palavras-chave:

Centros de Saúde; Programas de Rastreamento; Promoção da Saúde; Neoplasias; Conhecimento.

Keywords: Health Centers; Mass Screening; Health Promotion; Neoplasms; Knowledge.

Palabras clave: Centros de Salud; Tamizaje Masivo; Promoción de la Salud; Neoplasias; Conocimiento.

Submetido: $08 / 12 / 2019$

Aprovado: $15 / 05 / 2019$

Autor(a) para Correspondência: Lorena Alves Trajano R. Corina Dantas, 299 Derby Club - Sobral (CE) CEP: 62130-000 E-mail: loren.tj21@gmail.com

\section{RESUMO}

Este estudo avaliou o conhecimento dos médicos da atenção primária à saúde (APS) em Sobral-CE acerca das atuais recomendações para o rastreamento de câncer. Trata-se de estudo transversal, qualitativo, realizado com médicos da APS do município por meio de questionário que avaliou seu conhecimento sobre rastreamento dos tipos de câncer mais prevalentes no país. A análise das respostas se baseou nos consensos do Instituto Nacional do Câncer (INCA), da Organização Mundial da Saúde (OMS), da Sociedade Americana de Câncer (American Cancer Society - ACS) e da Força Tarefa Canadense em Assistência Preventiva à Saúde (Canadian Task Force on Preventive Health Care - (TFPHC). Os resultados indicam que a familiaridade com as diretrizes foi variável. Considerando os possiveis obstáculos observados na prevenção secundária, a preocupação com a falta de clareza nas recomendações para o rastreamento de câncer foi relatada por menos de $50 \%$ dos participantes, ao passo que os demais obstáculos foram citados por mais de 65\%. Concluiu-se ser necessário adotar esforços para aprimorar a educação médica em termos de prevenção secundária, com vistas a melhorar o diagnóstico precoce dos diversos tipos dessa doença.

1. Aluna de Graduação em Medicina na Universidade Federal do Ceará (UFC). Sobral (CE), Brasil. E-mail: Loren. tj21@gmail.com

2. Aluno de Graduação em Medicina na UFC. Sobral (CE), Brasil. E-mail: igorsqferreira@gmail.com

3. Aluno de Graduação em Medicina na UFC. Sobral (CE), Brasil. E-mail: migmarc2@gmail.com

4. Professor no Curso de Graduação em Medicina da UFC. Coordenador do Serviço de Oncologia e Hematologia da Santa Casa de Misericórdia de Sobral (SCMS). Sobral (CE),Brasil. E-mail: ledbatista@gmail.com 


\section{ABSTRACT}

This study assessed primary health care (PHC) physicians' knowledge in Sobral, Ceará, Brazil, on the current recommendations for cancer screening. This is a cross-sectional, qualitative, study carried out with PHC physicians in the municipality by means of a questionnaire that assessed their knowledge on screening of the most prevalent types of cancer in the country. The analysis of answers was based on consensuses of the National Cancer Institute (Instituto Nacional do Câncer - INCA), the World Health Organization (WHO), the American Cancer Society (ACS), and the Canadian Task Force on Preventive Health Care (CTFPHC). The results indicate that familiarity with the guidelines was variable. Considering the possible obstacles observed in secondary prevention, concern about the lack of clarity in recommendations for cancer screening was reported by less than 50\% of the participants, while the other obstacles were cited by more than 65\%. It was concluded that efforts are needed to improve medical education in terms of secondary prevention, with a view to improving the early diagnosis of various types of this disease.

\section{RESUMEN}

Este estudio evaluó el conocimiento de médicos de la atención primaria de salud (APS) en Sobral, Ceará, Brasil, acerca de las recomendaciones actuales para rastreo del cáncer. Este es un estudio transversal, cualitativo, realizado con médicos de la APS en el municipio mediante un cuestionario que evaluó su conocimiento sobre rastreo de los tipos de cáncer más prevalentes en el país. El análisis de respuestas se basó en los consensos del Instituto Nacional del Cáncer (Instituto Nacional do Câncer-INCA), de la Organización Mundial de la Salud (OMS), de la Sociedad Americana del Cáncer (American Cancer Society - ACS) y del Grupo de Trabajo Canadiense sobre el Cuidado Preventivo de Salud (Canadian Task Force on Preventive Health Care - CTFPHC). Los resultados indican que la familiaridad con las directrices fue variable. Teniendo en cuenta los posibles obstáculos observados en la prevención secundaria, la preocupación por la falta de claridad en las recomendaciones para rastreo del cáncer fue reportada por menos de $50 \%$ de los participantes, mientras que los otros obstáculos fueron citados por más de $65 \%$. Se concluyó que son necesarios esfuerzos para mejorar la educación médica en términos de prevención secundaria, con miras a mejorar el diagnóstico precoz de los varios tipos de esta enfermedad.

\section{INTRODUÇÃ 0}

As doenças não transmissiveis, como câncer, doenças cardiovasculares e diabetes mellitus, já superam as doenças infectocontagiosas no que concerne à incidência e à mortalidade em países de média renda, como o Brasil, principalmente em regiões que enfrentam problemas socioeconômicos, étnicos, geográficos e falta de acesso a serviços de saúde - a exemplo do Nordeste ${ }^{1,2}$.

No Brasil, segundo dados do Instituto Nacional do Câncer (INCA) estimados para 2016, excluindo os tipos de câncer de pele não melanoma, o câncer de mama é o de maior incidência entre as mulheres (cerca de 58 casos para 100.000 habitantes) e o câncer de próstata é o de maior incidência entre os homens (cerca de 61,2 casos para cada 100.000 habitantes). Em brasileiros do sexo masculino, a maior taxa de mortalidade se refere ao câncer de pulmão; já nas brasileiras, a mortalidade decorre, principalmente, do câncer de mama ${ }^{3}$. Para 2020, estima-se uma incidência de pouco mais de 500.000 casos de câncer no país (projeção de aumento de mais de 38\% em relação ao ano de 2009$)^{4}$.

Apresentando alguns dos piores índices socioeconômicos do país ${ }^{5}$, o Nordeste também acaba tendo dificuldades na prevenção e no diagnóstico de câncer. Quanto ao câncer de colo de útero, por exemplo, há dificuldade até para registrar a incidência da neoplasia nas áreas com os menores indices socioeconômicos, o que prejudica o acesso aos serviços voltados à prevenção, ao diagnóstico e ao tratamento ${ }^{6}$.

$\mathrm{Na}$ área oncológica, o diagnóstico precoce é uma estratégia que viabiliza terapias mais simples e efetivas, por contribuir para a redução do estágio de apresentação do câncer. Por essa razão, o conceito de diagnóstico precoce também é denominado downstaging. É importante que os profissionais da saúde reconheçam os sinais de alerta dos tipos de câncer mais comuns - passiveis de melhor prognóstico se descobertos no início?.

Basicamente, a prevenção primária de câncer consiste em evitar os fatores de risco que têm forte relação com tumores, como álcool, aspectos dietéticos, sedentarismo e exposição solar, sendo, 
por si, um meio efetivo que pode evitar de $1 / 3$ a $1 / 2$ dos casos de câncer. Já a prevenção secundária consiste no uso de métodos diagnósticos para detectar lesões predisponentes ao surgimento de câncer. Estudos mostram que a prevenção primária combinada à secundária é capaz de diminuir a morbimortalidade por câncer de colo de útero, além de reduzir em até $29 \%$ o número de mortes por câncer de mama ${ }^{8,9}$.

Atualmente, a indicação para o rastreamento está restrita aos casos de câncer de: a) mama; b) colo de útero; e c) cólon e reto. Entretanto, muitos outros tipos dessa doença, exceto câncer de pulmão e esôfago, são passíveis de diagnóstico precoce mediante avaliação e encaminhamento oportunos após os primeiros sinais e sintomas?

No Brasil, de acordo com o INCA, estima-se o surgimento de 600.000 novos casos no biênio 2016-2017, sendo 180.000 pacientes com câncer de pele não melanoma, logo, temos 420.000 novos casos de câncer propriamente ditos. Assim, o rastreamento precoce desses novos casos é crucial, com o propósito de intervir de modo incisivo desde o início, aumentando sobremaneira as chances de cura desses pacientes com diagnóstico oportuno ${ }^{3}$.

Um estudo semelhante foi realizado no Município de Sobral em 2005 - então se constatou que a maioria das recomendações dos médicos entrevistados não se adequava aos consensos adotados: as taxas de adequação a pelo menos um dos consensos variaram de 73,3\% (câncer de pele) a apenas 3,3\% (câncer de mama). Nesse estudo, em geral, as recomendações inadequadas mostravam nítida tendência ao excesso nos casos de câncer de colo de útero e de pulmão, e deficiência no rastreamento nos cânceres de mama e cólon e reto. Em relação às barreiras ao rastreamento adequado, $70 \%$ dos participantes consideraram de grande importância a falta de interesse do paciente e $66,6 \%$ apontaram a falta de educadores em saúde que atuam junto à população e a falta de verbas para custear os exames. Apesar da reconhecida importância desse tipo de estudo que aborda a aplicação das medidas de rastreamento por parte dos profissionais da saúde, a literatura nacional sobre o tema é escassa e a maioria dessas referências adotam pesquisas internacionais como base ${ }^{8}$.

Em razão disso, esta pesquisa avaliou 0 conhecimento dos médicos da atenção primária à saúde (APS) de Sobral sobre as recomendações mais atuais para o rastreamento de câncer, comparando os resultados obtidos aos de um estudo semelhante

\section{...rastreamento precoce desses novos casos é crucial, com o propósito de intervir de modo incisivo desde o início...}

realizado no ano de 2005, que não chegou a ser publicado, de modo a verificar se houve alguma evolução nestes últimos 15 anos, bem como quais deficiências ainda persistem, contribuindo para 0 planejamento, o desenvolvimento e a implementação de melhores estratégias para suprir as necessidades locais e sanar as deficiências existentes no norte do Ceará, sensibilizando lideranças governamentais e autoridades de saúde para buscar uma efetiva e constante melhoria dos serviços públicos.

Este estudo analisou, de modo qualitativo, o rastreamento dos casos de câncer na APS de Sobral executado pelos médicos - que buscam intervir positivamente tanto na vida dos usuários dos centros da Estratégia Saúde da Família (ESF) quanto no sistema de saúde como um todo, mediante a identificação de anormalidades em dois pontos principais: a) condutas de rastreamento de câncer; e b) pontos que prejudicam a execução desse rastreamento.

Dessa maneira, a pesquisa buscou identificar ações que precisam ser fortalecidas para aprimorar o rastreamento executado no âmbito da ESF, com o propósito de oferecer um serviço de saúde mais efetivo e eficar, diminuindo os números de novos casos de câncer a partir do reconhecimento de alterações pré-cancerígenas e de uma ação terapêutica adequada.

\section{METODOLOGIA}

Trata-se de estudo transversal qualitativo realizado com 30 médicos que atuam na APS de Sobral entre setembro 2016 e fevereiro de 2017; seu cenário consistiu em 17 centros da ESF. Vale salientar que 32 profissionais foram convidados a participar, mas 2 se recusaram.

As informações foram obtidas por meio de um questionário, intitulado "Médicos e Prevenção Secundária do Câncer", aplicado por acadêmicos do $4^{\circ}$ ao $11^{\circ}$ semestres do Curso de Graduação em 
Medicina da Universidade Federal do Ceará (UFC) campus de Sobral.

A abordagem aos médicos foi padronizada e os questionários foram preenchidos em unidades básicas de saúde (UBS), com a presença do aluno de graduação, sem consulta a qualquer bibliografia ou a terceiros por parte dos participantes.

Esse questionário coletou dados relativos a:

A. Idade e ano de graduação;

B. Condutas necessárias para rastrear os casos de câncer mais incidentes no Brasil (mama, colo de útero, próstata, cólon e reto e pele); e

C. Classificação dos obstáculos em "grande importância", "pouca importância" e "não considero uma barreira".

Alguns destes fatores foram enumerados (obstáculos 1 a 7 ):

i) Falta de agentes em promoção de saúde que orientem a população a procurar o médico;

ii) Falta de tempo do médico para realizar a prevenção secundária do câncer durante as consultas;

iii) Demora entre as consultas do mesmo paciente;

iv) Falta de interesse do paciente;

v) Falta de verba para custear os exames de prevenção; vi) Dificuldade para saber quando fazer o rastreamento; e

vii) Falta de conhecimento e/ou treinamento inadequado para empreender esse tipo de prevenção.

0 câncer de pulmão foi excluído do escopo deste estudo por conta de divergências quanto à eficácia de seu rastreamento ${ }^{7}$.

A análise das respostas se baseou nos consensos do INCA, da Organização Mundial da Saúde (OMS), da Sociedade Americana de Câncer (American Cancer Society - ACS) e da Força Tarefa Canadense em Assistência Preventiva à Saúde (Canadian Task Force on Preventive Health Care - (TFPHC).

Então, as condutas indicadas pelos entrevistados se classificaram em:

A. Adequada (quando os exames, a idade e a frequência indicados pelo entrevistado são exatamente 0 que preconiza 0 protocolo em comparação);

B. Inadequada deficiente (quando os exames recomendados pelo médico são insuficientes para um rastreamento satisfatório); e

C. Inadequada excessiva (que submete o paciente a estresse desnecessário, além de gerar custos adicionais ao sistema de saúde).

0 Quadro 1 sintetiza a classificação das respostas.

Quadro 1 - Classificação das respostas obtidas por meio dos questionários

\begin{tabular}{|c|c|c|c|}
\hline $\begin{array}{l}\text { Variáveis da conduta } \\
\text { indicada }\end{array}$ & Adequada & Inadequada deficiente & Inadequada excessiva \\
\hline Exames & $\begin{array}{l}\text { Idêntica ao } \\
\text { protocolo de } \\
\text { comparação. }\end{array}$ & $\begin{array}{c}\text { Pelo menos } 1 \text { dos exames } \\
\text { indicados no protocolo de } \\
\text { comparação não foi referido pelo } \\
\text { entrevistado. }\end{array}$ & $\begin{array}{l}0 \text { entrevistado referiu todos } \\
\text { os exames indicados pelo } \\
\text { protocolo de comparação e } \\
\text { algum(ns) outro(s) que não } \\
\text { consta(m) no protocolo. }\end{array}$ \\
\hline $\begin{array}{l}\text { Idade (de inicio/fim } \\
\text { de rastreamento) }\end{array}$ & $\begin{array}{l}\text { Idêntico ao } \\
\text { protocolo de } \\
\text { comparação. }\end{array}$ & $\begin{array}{l}0 \text { médico referiu idade de início } \\
\text { de rastreamento superior à } \\
\text { indicada pelo protocolo ou idade } \\
\text { em que rastreamento não deve } \\
\text { mais ser aplicado inferior à } \\
\text { preconizada. }\end{array}$ & $\begin{array}{l}0 \text { médico indicou início } \\
\text { precoce de rastreamento em } \\
\text { relação ao preconizado pelo } \\
\text { protocolo comparado ou fim do } \\
\text { rastreamento mais tardio do que } \\
\text { o recomendado. }\end{array}$ \\
\hline $\begin{array}{l}\text { Frequência (de } \\
\text { aplicação dos } \\
\text { exames) }\end{array}$ & $\begin{array}{l}\text { Idêntico ao } \\
\text { protocolo de } \\
\text { comparação. }\end{array}$ & $\begin{array}{l}0 \text { entrevistado indicou frequência } \\
\text { menor do que a preconizada } \\
\text { (o espaço de tempo entre um } \\
\text { exame e outro é maior do que o } \\
\text { recomendado). }\end{array}$ & $\begin{array}{l}\text { O entrevistado indicou } \\
\text { frequência maior do que a } \\
\text { preconizada (o espaço de tempo } \\
\text { entre um exame e outro é menor } \\
\text { do que o recomendado). }\end{array}$ \\
\hline
\end{tabular}

Fonte: Elaborado pelos autores.

Exames indicados pelos médicos para grupos de risco ou pacientes sintomáticos foram considerados procedimento de vigilância e não rastreamento, assim, essas respostas configuraram ausência de indicação de rastreamento pelo entrevistado. Já os médicos que deixaram de responder questões referentes às suas indicações para rastreamento de determinado tipo de câncer corresponderam à classificação “conduta 
inadequada deficiente". Adotou-se tal medida com base na hipótese de que o médico que não respondeu a uma questão específica, mesmo tendo notificado que a deixaria em branco, não tinha conhecimento sobre o que se recomenda para o rastreamento daquele tipo de câncer, não apresentando, portanto, nem uma conduta adequada nem uma conduta inadequada excessiva.

Não foi necessária a aplicação de testes estatísticos para o cálculo da amostra, uma vez que este estudo abrangeu um número próximo da totalidade dos médicos da APS de Sobral.

A pesquisa obteve aprovação do Comitê de Ética em Pesquisa, da Universidade Estadual Vale do Acaraú (UVA), sob o Parecer n. 3.056.527/2018, em consonância com a Resolução CNS n. 466/2012.

\section{RESULTADOS E DISCUSSÃO}

A partir dos dados coletados na primeira parte do questionário, avaliou-se o grau de informação dos profissionais da saúde que participaram do estudo sobre as práticas para rastreamento e diagnóstico precoce dos tipos de câncer com maior incidência em nosso país (mama, colo de útero, próstata, cólon e reto e pele). As recomendações dos principais consensos utilizados para o rastreamento de câncer são sintetizadas no Quadro 2.

Quadro 2 - Medidas de rastreamento por tipo de câncer preconizadas por cada consenso

\begin{tabular}{|c|c|c|c|c|c|c|c|c|}
\hline \multirow[b]{2}{*}{ Método } & \multicolumn{2}{|c|}{ INCA } & \multicolumn{2}{|c|}{ OMS } & \multicolumn{2}{|c|}{ СTFPHC } & \multicolumn{2}{|c|}{ ACS } \\
\hline & $\begin{array}{l}\text { Idade } \\
\text { (anos) }\end{array}$ & Frequência & $\begin{array}{l}\text { Idade } \\
\text { (anos) }\end{array}$ & Frequência & $\begin{array}{l}\text { Idade } \\
\text { (anos) }\end{array}$ & Frequência & $\begin{array}{l}\text { Idade } \\
\text { (anos) }\end{array}$ & Frequência \\
\hline \multicolumn{9}{|c|}{ Mama } \\
\hline Exame clínico & $>40$ & Anual & $\begin{array}{c}\text { Não } \\
\text { indica }\end{array}$ & Não indica & $>50$ & Anual & $>20$ & $\begin{array}{l}\text { Trienal para } \\
20-40 \text { anos } \\
\text { e anual para } \\
>40 \text { anos }\end{array}$ \\
\hline Autoexame & Não indica & Não indica & $\begin{array}{c}\text { Não } \\
\text { indica }\end{array}$ & Não indica & Não indica & Não indica & $\begin{array}{c}20 \\
\text { (opcional) }\end{array}$ & Opcional \\
\hline Mamografia & $>50$ & $\begin{array}{l}\text { Bienal, no } \\
\text { máximo }\end{array}$ & $>50$ & $\begin{array}{c}\text { A cada } 2 \text { ou } \\
3 \text { anos }\end{array}$ & $>50$ & Anual & $>40$ & Anual \\
\hline \multicolumn{9}{|c|}{ Colo de útero } \\
\hline Papanicolau & $\begin{array}{c}\text { Após } \\
\text { início da } \\
\text { atividade } \\
\text { sexual ou } \\
>25\end{array}$ & Anual & $>35$ & $\begin{array}{l}\text { Não refere } \\
\text { frequência }\end{array}$ & $\begin{array}{c}\text { Após } \\
\text { início da } \\
\text { atividade } \\
\text { sexual ou } \\
>18\end{array}$ & Anual & $\begin{array}{c}>21 \text { ou } 3 \\
\text { anos após } \\
\text { sexarca }\end{array}$ & Anual \\
\hline \multicolumn{9}{|c|}{ Próstata } \\
\hline $\begin{array}{l}\text { Antígeno } \\
\text { prostático } \\
\text { específico e } \\
\text { toque retal }\end{array}$ & Não indica & Não indica & $\begin{array}{l}\text { Indica } \\
\text { sem } \\
\text { referir } \\
\text { idade }\end{array}$ & $\begin{array}{l}\text { Indica sem } \\
\text { referir } \\
\text { idade }\end{array}$ & Não indica & Não indica & $>50$ & Anual \\
\hline \multicolumn{9}{|c|}{ Cólon e reto } \\
\hline Sangue oculto & $>50$ & Anual & $\begin{array}{c}\text { Não } \\
\text { indica }\end{array}$ & Não indica & $>50$ & $\begin{array}{c}\text { Máximo } 2 \\
\text { anos }\end{array}$ & $>50$ & Anual \\
\hline Colonoscopia & Não indica & Não indica & $\begin{array}{c}\text { Não } \\
\text { indica }\end{array}$ & Não indica & Não indica & $\begin{array}{c}\text { Máximo } 2 \\
\text { anos }\end{array}$ & $>50$ & $\begin{array}{c}\text { A cada } 10 \\
\text { anos }\end{array}$ \\
\hline \multicolumn{9}{|c|}{ Pele } \\
\hline Exame clínico & Não indica & Não indica & $\begin{array}{l}\text { Indica } \\
\text { sem } \\
\text { referir } \\
\text { idade }\end{array}$ & $\begin{array}{l}\text { Indica sem } \\
\text { referir } \\
\text { frequência }\end{array}$ & Não indica & Não indica & $>20$ & Ocasional \\
\hline
\end{tabular}

Fonte: Elaborado pelos autores. 
0s dados da Tabela 1 foram obtidos mediante classificação das condutas descritas. Constatou-se a adoção de conduta adequada em mais de $50 \%$ dos casos apenas em relação ao câncer de mama - achado similar ao de outro estudo realizado nos Estados Unidos da América (EUA) ${ }^{10}$. Entretanto, não se pode considerar tal índice um sinal de qualidade do atendimento, pois essa malignidade é a de maior incidência e mortalidade entre as mulheres, portanto, seu rastreamento deveria abranger toda a população indicada pelos consensos.

Tabela 1 - Índice de adequação das condutas indicadas pelos médicos para rastreamento dos principais tipos de câncer

\begin{tabular}{lccc}
\hline Tipo de câncer & Conduta adequada N (\%) & $\begin{array}{c}\text { Conduta inadequada } \\
\text { excessiva N (\%) }\end{array}$ & $\begin{array}{c}\text { Conduta inadequada } \\
\text { deficiente N (\%) }\end{array}$ \\
\hline Mama & $16(53,3 \%)$ & $9(30 \%)$ & $5(16,6 \%)$ \\
Colo de útero & $12(40 \%)$ & $6(20 \%)$ & $12(40 \%)$ \\
\hline Próstata & $3(10 \%)$ & $24(80 \%)$ & $3(10 \%)$ \\
Cólon e reto & $5(16,6 \%)$ & $6(20 \%)$ & $19(63,3 \%)$ \\
\hline Pele & $4(13,3 \%)$ & $14(46,6 \%)$ & $12(40 \%)$ \\
\hline
\end{tabular}

Fonte: Elaborada pelos autores.

Nos demais casos, chamam atenção o câncer de pele $(13,3 \%$ dos profissionais apontaram a conduta adequada), e as neoplasias de colo de útero e cólon e reto (nas quais houve tomada de conduta inadequada deficiente em $40 \%$ e $63 \%$ dos casos, respectivamente). Em tal situação, deve-se salientar que essas 3 estão entre as malignidades mais incidentes em nosso meio, justificando a necessidade dos profissionais da saúde atentarem ao rastreamento e diagnóstico precoce delas. No caso do câncer de colo de útero, por exemplo, o qual se comporta como malignidade de progressão lenta, com longo período de prevenção eficaz no sexo feminino, a conduta adequada foi adotada em apenas $40 \%$ dos casos, indicando elevado déficit de conhecimento dos profissionais sobre uma enfermidade relevante em nosso meio. Inclusive, um estudo realizado em Boa Vista-RR identificou que, apesar do déficit no rastreamento correto para outros tipos de câncer, o de colo de útero teve recomendação de condutas adequadas em $95,7 \%$ dos $\operatorname{casos}^{11}$. Deve-se investigar os motivos para tais diferenças em diferentes locais quanto à indicação do Papanicolau - exame realizado com frequência que deve ser dominado por todos os médicos da APS.

A detecção precoce de câncer pode salvar vidas, reduzir a morbidade associada ao curso da doença e diminuir os custos do tratamento das doenças no sistema de saúde. Ela deve ser estruturada na atenção à saúde, com clara definição de suas estratégias e efetiva incorporação de seus princípios técnicos e operacionais por parte dos profissionais da saúde. Segundo Aguiar et al. ${ }^{12}$, a efetividade do cuidado depende da reorganização dos processos de trabalho, observando-se a importância do médico realizar o diagnóstico nas fases iniciais para que os pacientes tenham encaminhamento efetivo aos serviços secundários e terciários.

A conduta inadequada excessiva foi observada, principalmente, no câncer de próstata $(80 \%)$, mas, ainda, no câncer de pele (quase $50 \%$ dos casos). Esse tipo de conduta também merece destaque, pois a intenção original da triagem é detectar o câncer nos estágios iniciais para melhorar os resultados de diagnóstico e tratamento e aumentar a sobrevida dos pacientes. Porém, quando há diagnóstico excessivo, definido como “diagnóstico de uma condição que não iria causar sintomas ou dano adicional, se não tratada, ao paciente durante o seu tempo de vida"13, o rastreamento não se torna custo-efetivo e pode trazer mais danos, por conta de procedimentos desnecessários, do que benefícios. Embora nenhum médico tenha a intenção de "tratar com excessos" ou "diagnosticar em excesso" o câncer, as triagens e a conscientização dos pacientes têm aumentado a chance de identificar um espectro de tipos de câncer, alguns dos quais não fatais e cujo diagnóstico precoce não altera a sobrevida.

Em relação ao mesmo estudo, também realizado em Sobral no ano de 2005, a taxa de adequação a pelo menos um consenso no caso do câncer de mama foi de 3,3\%; quanto ao câncer de pele, essa taxa foi de $73,3 \%$, com expressiva evolução no primeiro caso se contrapondo a relevante queda do índice no segundo. No geral, a maioria das recomendações não estava adequada aos consensos adotados, revelando 
um déficit permanente após 12 anos.

No interior dos EUA se realizou um estudo similar sobre o conhecimento dos médicos a respeito das recomendações da ACS quanto ao rastreamento e ao diagnóstico precoce de câncer e constatou-se que $70 \%$ dos médicos afirmam ter conhecimento dessas medidas. Entretanto, $77 \%$ consideram a educação médica insuficiente para proporcionar medidas de prevenção e $43 \%$ afirmam que as recomendações não são claras, de modo que $17 \%$ responderam incorretamente questões sobre as medidas de prevenção do câncer de cólon e reto. Ademais, $68 \%$ dos médicos acreditam que as recomendações para câncer de próstata consistam apenas na dosagem de antígeno prostático específico (prostale specific antigen - PSA), entre outras medidas que não preconizadas pela $\mathrm{ACS}^{14}$, o que sugere insuficiente conhecimento dos médicos sobre prevenção e rastreamento de câncer não só em nosso meio.

Mostra-se necessário aumentar a adesão dos médicos aos consensos, o que demanda recursos e esforços educativos. Entendemos ser indispensável um esforço multidisciplinar que enfatize a importância das medidas preventivas na educação médica. A Tabela 2 sintetiza os obstáculos descritos que constituíram a terceira parte do questionário aplicado, classificados em grande importância, pouca importância e não considero barreira.

Tabela 2 - Respostas da terceira parte do questionário aplicado

\begin{tabular}{lccc}
\hline \multirow{2}{*}{ Obstáculos } & \multicolumn{2}{c}{ Grau de importância apontado pelos médicos - N (\%) } \\
\cline { 2 - 4 } & Grande importância & Pouca importância & Não considero barreira \\
\hline Obstáculo 1 & $20(72 \%)$ & $10(14 \%)$ & $10(14 \%)$ \\
\hline Obstáculo 2 & $24(82 \%)$ & $6(14 \%)$ & $0(0 \%)$ \\
\hline Obstáculo 3 & $20(66 \%)$ & $9(32 \%)$ & $3(6 \%)$ \\
\hline Obstáculo 4 & $23(76 \%)$ & $4(18 \%)$ & $4(7 \%)$ \\
\hline Obstáculo 5 & $22(74 \%)$ & $4(19 \%)$ & $7(26 \%)$ \\
\hline Obstáculo 6 & $13(42 \%)$ & $9(32 \%)$ & $3(12 \%)$ \\
\hline Obstáculo 7 & $19(64 \%)$ & $7(24 \%)$ & $3(6)$ \\
\hline
\end{tabular}

Obstáculo 1: falta de agentes em promoção de saúde que orientem a população a procurar o médico.

Obstáculo 2: falta de tempo do médico para realizar a prevenção secundária de câncer durante as consultas.

Obstáculo 3: demora entre as consultas do mesmo paciente.

Obstáculo 4: falta de interesse do paciente.

Obstáculo 5: falta de verba para custear os exames de prevenção.

Obstáculo 6: dificuldade em saber quando fazer o rastreamento.

Obstáculo 7: pouco conhecimento e/ou treinamento inadequado para fazer esse tipo de prevenção.

Fonte: Elaborada pelos autores.

Assim, observa-se que, exceto no obstáculo 6, todos foram avaliados como de grande importância por mais de $50 \%$ dos participantes - um resultado positivo, pois esse pode ser o primeiro passo para vencer tais obstáculos, e aumentar o conhecimento e melhorar o trabalho dos profissionais da saúde no diagnóstico precoce de câncer.

0 fato das "recomendações para fazer rastreamento dessas doenças não serem muito claras" terem sido apontadas apenas por $42 \%$ dos profissionais como de grande importância indica que não há clareza quanto às recomendações dos consensos para o rastreamento e diagnóstico precoce. 0 déficit nesse aspecto, somado à falta de conhecimento dos médicos diante dessas recomendações, leva-nos a concluir que a melhoria dessa situação certamente resultaria na obtenção de bons resultados no campo da saúde.

Além desses fatores, outro aspecto relacionado à dificuldade dos profissionais para aderir às recomendações seria a própria alteração frequente nas diretrizes. Um estudo realizado com ginecologistas/ obstetras americanos, por exemplo, demonstrou que inclusive especialistas na área apresentaram dificuldade para acompanhar as mudanças de recomendações nas diretrizes de rastreamento para o câncer de mama ${ }^{15}$.

As demais barreiras foram referidas como de grande importância, a exemplo da falta de educadores em saúde que estimulem a procura dos médicos pela população, bem como a falta de interesse do paciente, são fatores relativos à própria população que dificultam sobremaneira a adequada prestação 
de serviços por parte dos médicos. Em outro estudo, realizado em 2005 na mesma cidade, 70\% dos participantes consideraram de grande importância a falta de interesse do paciente e $66,6 \%$ a falta de educadores em saúde. Esse estudo também apontou que $66,6 \%$ indicaram ser importante a falta de verba para custear exames, resultado permanente em nosso estudo (importante para $74 \%$ dos participantes).

Vale notar, ainda, que $64 \%$ dos médicos apontaram como de grande importância a falta de conhecimento e/ou treinamento adequado para proporcionar esse tipo de prevenção. Tais resultados evidenciam que os médicos reconhecem sua falta de conhecimento sobre as medidas corretas para prevenção de câncer e sentem dificuldade diante da falta de treinamento prévio que enfoque essa questão.

\section{CONCLUSÃO}

A partir dos dados discutidos, constata-se um elevado déficit de conhecimento que poderia melhorar os índices de mortalidade de uma doença tão relevante como o câncer em nosso meio. De modo geral, os médicos não dominam as medidas preconizadas pelos consensos adotados - isso deve mudar, em prol da saúde pública de nosso país.

Também se evidenciou interesse dos médicos no diagnóstico precoce e na prevenção das condições mais prevalentes em nosso meio, porém, eles apresentam dificuldade para seguir o que é preconizado pelos consensos de prevenção, seja por conta da não homogeneidade de tais consensos ou pela própria falta de conhecimento sobre eles. Concluímos que nosso meio demanda um esforço multidisciplinar com foco na medicina preventiva, opção de escolha no âmbito da APS, enfatizando a importância das medidas de rastreamento para a prevenção de doenças de extrema relevância no país.

Percebe-se, ainda, acentuada escassez de literatura sobre o tema no Brasil, lacuna que deve ser

\section{Percebe-se, ainda, acentuada escassez de literatura sobre$$
\text { o tema no }
$$$$
\text { Brasil... }
$$

superada para avaliar adequadamente o conhecimento dos médicos sobre medidas de rastreamento de câncer em diversas localidades, com vistas a prover subsídios para a evolução da educação médica e, consequentemente, a qualificação dos serviços de saúde nacionais.

\section{CONTRIBUIÇÃO DOS AUTORES}

Lorena Alves Trajano, José Francisco Igor Siqueira Ferreira, Miguel Marcelo Freire de Melo e Luiz Eduardo de Castro Batista contribuíram com a realização da pesquisa, o delineamento do estudo e a redação e revisão crítica do manuscrito.

\section{REFERÊNCIAS}

1. Organização Pan-Americana da Saúde. Condições de saúde e suas tendências. In: OPAS. Saúde nas Américas. Brasília (DF): OPAS; 2007. v. 1, p. 62-217. (Publicação Científica e Técnica OPAS n. 622).

2. Duncan BB, Chor D, Aquino EML, Bensenor IM, Mill JG, Schmidt MI, et al. Doenças crônicas não transmissiveis no Brasil: prioridade para enfrentamento e investigação. Rev Saúde Pública [serial on the internet]. 2012 [cited 2019 May 24];46(Suppl):126-34. Available from: http://www. scielo.br/pdf/rsp/v46s1/17.pdf

3. Brasil. Estimativa 2016: incidência de câncer no Brasil. Rio de Janeiro: Instituto Nacional de Câncer; 2015.

4. Goss PE, Lee BL, Badovinac-Cmjevic T, StrasserWeippl J, Chavarri-Guerra Y, St Louis J, et al. Planejamento do controle do câncer na América Latina e no Caribe. Lancet Oncol. 2013;14:391-436.

5. Instituto de Pesquisa Econômica Aplicada. Atlas do desenvolvimento humano no Brasil [document on the internet]. date unknown [cited on 8 Sep 2017]. Available from: http://www.atlasbrasil.org. $\mathrm{br} / 2013 / \mathrm{pt} / \mathrm{consulta/}$

6. Gamarra CJ, Valente JG, Silva GA. Magnitude da mortalidade por câncer do colo do útero na Região Nordeste do Brasil e fatores socioeconômicos. Rev Panam Salud Publica [serial on the internet]. 2010 [cited 2019 May 24];28(2):100-6. Available from: https://scielosp.org/pdf/rpsp/2010.v28n2/100$106 / p t$

7. Brasil. Caderno de atenção primária: rastreamento. Brasília (DF): Ministério da Saúde; 2010. 
8. Tucunduva LTCM, Sá VHLC, Koshimura ET, Prudente FVB, Santos AF, Samano EST, et al. Estudo da atitude e do conhecimento dos médicos não oncologistas em relação às medidas de prevenção e rastreamento do câncer. Rev Assoc Méd Bras [serial on the internet]. 2004 [cited 2019 May 24];50(3):257-62. Available from: http://www.scielo.br/pdf/ramb/ v50n3/21655.pdf

9. Vineis P, Wild CP. Global cancer patterns: causes and prevention. Lancet. 2014;(383):549-57.

10. Hinz EK, Kudesia R, Rolston R, Caputo TA, Worley MJ Jr. Physician knowledge of and adherence to the revised breast cancer screening guidelines by the United States Preventive Services Task Force. Am J Obstet Gynecol. 2011;(205):e1-e5.

11. Silva LMC, Fonseca AJ, Ferreira LP, Dalla-Benetta $A C$, Navarro C. Atitude e conhecimento de médicos da Estratégia Saúde da Família sobre prevenção e rastreamento do câncer. Rev Bras Cancerol [serial on the internet]. 2011 [cited 2019 May 24];57(4):52534. Available from: http://www1.inca.gov.br/ $\mathrm{rbc/n} 57 / \mathrm{v} 04 / \mathrm{pdf} / 09$ artigo atitude e conhecimento medicos estrategia saude familia sobre prevencao rastreamento cancer.pdf

12. Aguiar FAR, Sousa TC, Branco JG0, Costa FBC, Torres ARA, Arruda LP. Produção do cuidado na rede de atenção ao câncer de mama: revisão integrativa. Sanare (Sobral, Online) [serial on the internet]. 2018;17(1):84-92. Available from: https:// sanare.emnuvens.com.br/sanare/article/ view $/ 1226 / 657$

13. Kale MS, Korenstein D. Overdiagnosis in primary care: framing the problem and finding solutions. BMJ. 2018;(362):k2820.

14. Ashford A, Gemson D, Gorin SNS, Bloch S, Lantigua $R$, Ahsan $H$, et al. Cancer screening and prevention practices of inner-city physicians. Am J Prev Med. $2000 ;(19): 59-62$.

15. Anderson BL, Pearlman M, Griffin J, Schulkin J. Conflicting and changing breast cancer screening recommendations: survey study of a national sample of ob-gyns after the release of the 2009 USPSTF guidelines. J Healthc Qual Res. 2013;35(4):25-35.
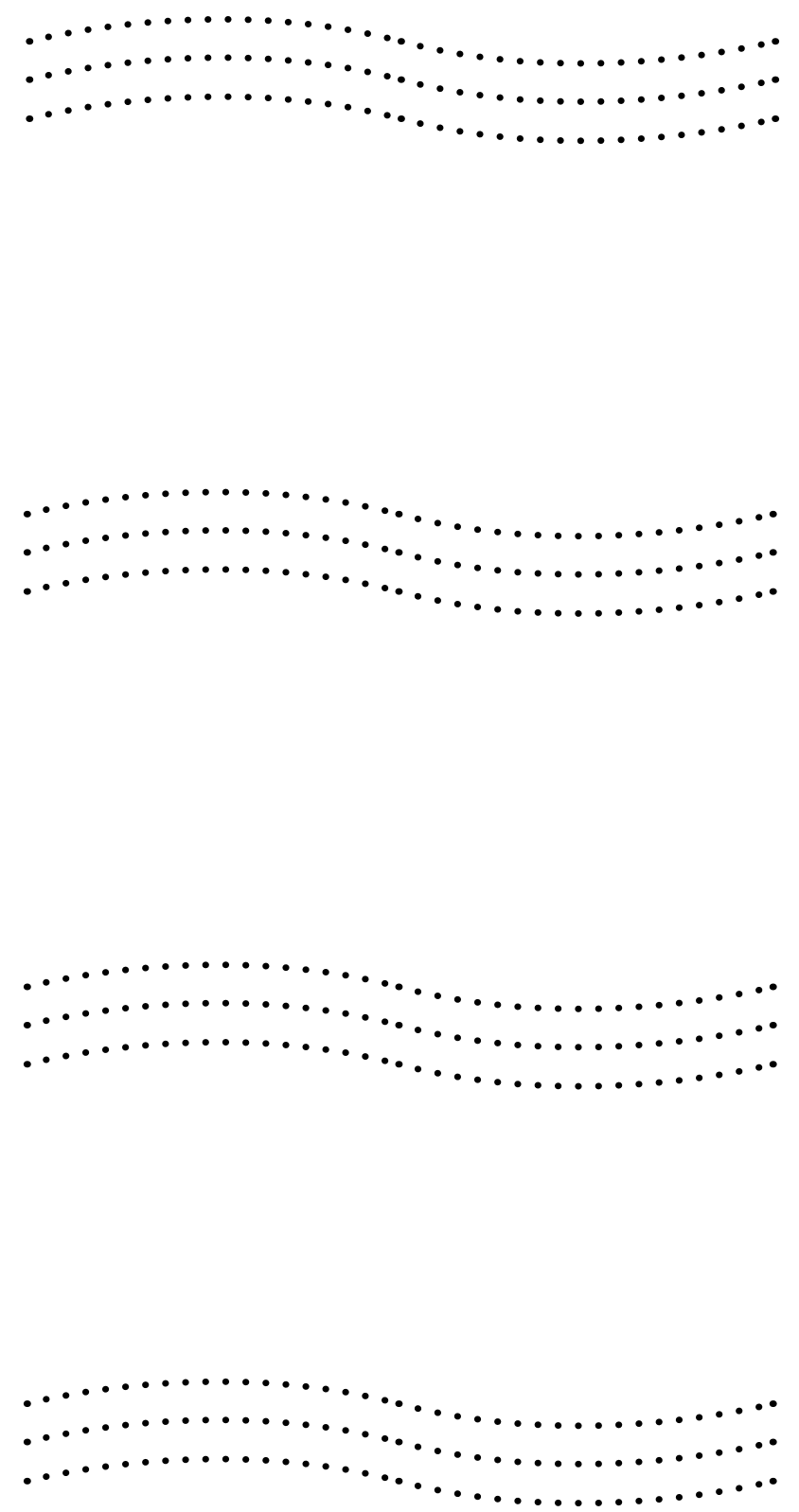

$\ldots \ldots \ldots \ldots \ldots \ldots \ldots \ldots \ldots \ldots \ldots \ldots \ldots \ldots \ldots \ldots$

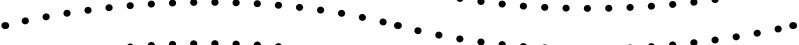

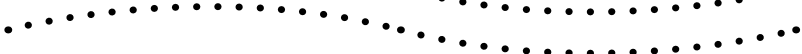

